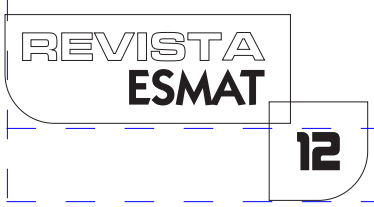

\title{
LA PROTECTION DES LIBERTES ET DROITS FONDAMENTAUX PAR LE JUGE DANS LES PHASES PREPARATOIRES DU PROCES PENAL RUSSE
}

THE JUDICIAL PROTECTION OF LIBERTIES AND FUNDAMENTAL RIGHTS IN THE PRELIMINARY STAGES OF RUSSIAN CRIMINAL PROCEDURE

Leonid Golovko

Professeur à la Faculté de Droit de l'Université d'Etat de Moscou (Lomonossov), directeur du Centre de procédure pénale, de justice et d'organisation du ministère public.

\section{RESUME}

Les actes fondamentaux relatifs à la protection des droits de l'homme, tels que la Déclaration universelle de 1948 ou le Pacte international de 1966, n'imposent aucun modèle de protection judiciaire de ces droits lors des phases préparatoires de la procédure pénale. Pourtant, l'évolution constitutionnelle ultérieure dans différents pays du monde, notamment en Russie, a fait émerger certains standards au regard desquels seul le juge peut limiter les libertés et droits fondamentaux dans les phases pre-judiciaires du procès pénal. Mais qui est ce juge? C'est un juge qui mène l'instruction (modèle français du juge d'instruction) ou c'est un juge extérieur à l'instruction? Dans ce dernier cas, s'agit-il du juge ordinaire ou d'un juge spécialisé? Le droit russe hésite toujours entre ces modèles. Ces hésitations traduisent certains problèmes conceptuels concernant le rôle du juge lors des phases préparatoires du procès pénal. Doit-il être considéré comme un instrument efficace de la protection des droits de l'homme ou plutôt comme un instrument de simple légitimation des actes non-judiciares limitant les libertés et les droits fondamentaux?

MOTS-CLES: Procédure pénale; droits de l'homme; juge; instruction préparatoire; légitimation

\section{ABSTRACT}

The core international human rights treaties, such a Universal Declaration of Human Rights of 1948 or International Covenant on Civil and Political Rights of 1966, do not 
establish any model of judicial protection of these rights during the preliminary stages of criminal procedure. However, the further constitutional evolution of different countries, in particular of Russia, created some universal standards. In accordance with these standards, it is only the judge may limit the freedoms and fundamentals rights in prejudicial stages of criminal procedure. Nevertheless, who is this judge? A judge who investigate (the French model of juge d'instruction) or a judge who is outside of investigation? In the latter case, it shall be an ordinary judge or a specialized judge? The Russian law yet hesitates between all these models. These hesitations are reflecting some conceptual problems concerning the role of judge in the preliminary stages of criminal procedure. Is the judicial activity shall be considering like an efficient instrument of protection of human rights or rather like an instrument of simple legitimation of nonjudicial acts limiting the freedoms and fundamental rights?

KEYWORDS: Criminal procedure; human rights; judge; preliminary investigation; legitimation.

\section{INTRODUCTION}

Lorsque l'on analyse les dispositions des actes fondamentaux sur les droits de I'homme, tels que la Déclaration universelle des droits de l'homme de 1948 ou le Pacte international relatif aux droits civils et politiques de 1966, dans le contexte du problème qui nous intéresse, c'est-à-dire la protection par le juge des libertés et droits fondamentaux dans les phases préparatoires du procès pénal, il devient rapidement clair qu'elles ne sont pas nombreuses et sont assez flous en la matière. On peut mentionner l'art. 8 de la Déclaration universelle: «Toute personne a droit à un recours effectif devant les juridictions compétentes contre les actes violant les droits fondamentaux qui lui sont reconnus par la constitution ou par la loi». Même si les phases préparatoires du procès pénal ne sont pas ici directement évoquées, elles sont bien évidemment sous-jacentes. On peut mentionner également l'art. 9 (al. 3) du Pacte international: «Tout individu arrêté ou detenu du chef d'une infraction pénale sera traduit dans le plus court délai devant un juge ou une autre autorité habilitée par la loi à exercer des fonctions judiciaires, et devra être jugé dans un délai raisonnable ou libéré».

C'est tout. Le reste (l'art. I0 de la Déclaration universelle, l'art. I4 du Pacte international etc.) touche d'autres aspects relatifs à la protection des droits de l'homme et des libertés individuelles lors de la procédure pénale: soit ceux qui concernent la phase du jugement, soit ceux qui concernent d'autres droits fondamentaux, tels que, par exemple, le droit à l'avocat. Mais même les dispositions citées de l'art. 8 de la Déclaration et de l'art. 9 du Pacte ne semblent pas être très concrètes, traduisant au niveau du modèle proposé plutôt un compromis politique, voire géopolitique, entre différents 
systèmes juridiques des Etats qui se sont opposés pendant la guerre froide.

Néanmoins, lorsque l'on analyse les dispositions des différentes Constitutions modernes, il est possible de réaliser dans quelle mesure le droit a évolué depuis 1948 et même depuis 1966. Autrement dit, le droit constitutionnel contemporain se présente comme beaucoup plus exigeant, au moins sur le plan normatif, que le droit international fondateur des droits de l'homme en ce qui concerne la protection par le juge des libertés et droits fondamentaux dans les phases préparatoires du procès pénal. Ainsi, par exemple, la Constitution russe de 1993 prévoit en la matière de nombreuses dispositions qui sont, de surcroît, assez concrètes. Citons en quelques unes :

- la détention provisoire ne peut être ordonnée que par le juge (art. 22);

- la limitation du droit au secret de la correspondance, des entretiens téléphoniques, des communications postales, télégraphiques et autres n'est permise que sur la base d'une décision judiciaire (art. 23);

- la pénétration dans un domicile contre la volonté des personnes qui y habitent ne peut avoir lieu que sur la base d'une décision judiciaire (art. 25).

II est clair que toutes ces dispositions constitutionnelles russes rendent l'intervention du juge dans les phases préparatoires du procès pénal absolument indispensable, faute de quoi il est impossible de procéder à la perquisition au domicile ou à l'interception des communications téléphoniques de la personne poursuivie pénalement, sans même parler de sa détention provisoire. Et cette liste des droits protégés par la Constitution russe qui nécéssitent l'intervention du juge au cours des phases préparatoires de la procédure pénale n'est pas exhaustive. On n'a donné que quelques exemples, même s'ils sont, semble-t-il, les plus importants.

II est certain qu'il ne s'agit évidemment pas d'une particularité du droit russe. La même situation peut être observée dans la majorité des pays, indépendamment de leurs traditions juridiques et culturelles. De là découle un principe fondamental et aujourd'hui universellement (ou quasi-universellement) reconnu - le principe dont les racines se retrouvent dans la Déclaration de 1948 et le Pacte de 1966 et qui a été développé ultérieurement par l'évolution du droit international et des droits nationaux, surtout au niveau constitutionnel: seul le juge peut limiter les libertés et droits fondamentaux dans les phases préparatoires du procès pénal.

Autrement dit, si la limitation de certains droits est indispensable pour le fonctionnement efficace de la justice pénale de n'importe quel pays (elle ne peut pas fonctionner sans détention provisoire, perquisitions, écoutes téléphoniques, etc.), ce n'est que le juge, avec son statut particulier (indépendant, impartial, etc.) qui peut légitimer une telle limitation des droits fondamentaux. II s'agit, en quelque sorte, d'un

'Sur le plan comparé, v. par exemple: PRADEL, Jean. Droit pénal comparé. 4 éd. Paris: Dalloz. 2016. P. 399, 466 et autres. 
axiome pour le droit contemporain, dans le sens mathématique du terme «axiome» signifiant une disposition qui n'a plus besoin d'être prouvée, même si les juristes préférent parler d'un grand principe ou d'un principe fondamental.

Pourtant, la réalisation technique de ce principe dans le système processuel d'un pays concerné pose des problèmes purement juridiques. On va essayer d'analyser certains de ces problèmes à travers bien évidemment l'exemple russe, placé dans le contexte du droit comparé.

\section{LE JUGE D'INSTRUCTION (LE JUGE QUI INSTRUIT) OU LE JUGE DE L'INSTRUCTION (LE JUGE QUI CONTROLE)?}

Le système classique, tel qu'il a été imaginé en France et réalisé dans le fameux Code d'instruction criminelle de I808, se base sur l'idée que l'instruction préparatoire ne peut être que judiciaire, c'est-à-dire un «organe central de l'instruction, le juge d'instruction exerce... la fonction d'instruction, au sens qu'il est d'abord un enquêteur; mais il a aussi des pouvoirs juridictionnels, étant une véritable juridiction du premier degré, une juridiction d'instruction» (GUINCHARD et BUISSON, p. 567). En d'autres termes, celui qui instruit (le juge d'instruction), «est un juge du tribunal de grande instance, c'est-à-dire un magistrat du siège inamovible et irrévocable... nommé dans ces fonctions dans les formes prévues pour les magistrats du siège...»(VINCENT et autres, p. 4 I3).

De là résulte que si c'est le juge (indépendant et impartial) qui mène l'instruction, il est en même temps d'office tout à fait légitime à limiter les droits fondamentaux de la personne au cours des phases préparatoires du procès pénal, c'est-à-dire d'ordonner la détention provisoire, procéder aux perquisitions et aux saisies, ordonner l'interception des conversations téléphoniques, etc. En ce sens, il est possible de constater que la fonction d'instruction judiciaire, couvrant un domaine plus large, inclut en quelques sorte automatiquement la fonction du juge de protéger les libertés et droits fondamentaux.

II s'agit du système classique pour l'Europe continentale dit du «juge d'instruction». Dans ce système, en ce qui concerne l'instruction préparatoire, le problème de l'intervention judiciaire dans les phases préparatoires du procès pénal ne se pose même pas, car l'instruction est judiciaire ex natura sua (par sa nature). Ce problème existe uniquement dans le cadre de l'enquête policière, complètement marginalisée par le Code d'instruction criminelle (sauf l'enquête in flagranti), mais tout naturellement développée par la pratique ultérieure et les nouvelles codifications en la matière (en France, par exemple, par le Code de procédure pénale de 1958).

Revenant à la Russie, la procédure pénale russe a également connu le système du juge d'instruction en 1864, où il a été introduit avec la Grande réforme judiciaire d'Alexandre II (par exemple, FOINITSKY, p. 370, 372), et jusqu'à la période soviétique, lorsqu'il a été définitivement supprimé, non sans certaines hésitations, par le nouveau pouvoir avec les codifications de procédure pénale des années 20 , survenues après la 
Révolution de 1917. S'agissant du système soviétique, l'instruction préparatoire a été maintenue, mais confiée soit au Ministère public (procuratura soviétique) pour les affaires les plus importantes, soit aux agents d'instruction du ministère de l'intérieur, toujours sous tutelle du procureur (par exemple, STROGOVITCH, p. 97). C'est le procureur qui légitimait la limitation des droits et libertés dans les phases préparatoires du procès pénal soviétique.

Avec la chute de l'URSS et même avant encore l'adoption de la Constitution de 1993, les débats sur la restauration du juge d'instruction à la française (ou plutôt à la russe, version 1864) ont été lancé dans la doctrine (par exemple, TROUSSOV, p. 62). A vrai dire, ces débats continuent toujours. Mais le retour de la Russie au système des juges d'instruction est peu probable, au moins pour l'instant.

En premier lieu, le législateur a préféré instituer un Comité d'instruction de la Fédération de Russie, une autorité indépendante, mais non-judiciaire et dont la structure intérieure est hiérarchisée. Si le nouveau Code de procédure pénale (CPP) russe, entré en vigueur le ler juillet 2002, a preservé le système soviétique dans lequel le procureur jouait un rôle central dans les phases préparatoires (enquête et instruction préparatoire) du procès pénal, c'est la loi du 5 juin 2007 qui a d'abord renforcé l'autonomie des « organes d'instruction » du parquet au sein de ce dernier, sans, à ce moment, complètement couper le cordon ombilical et sans toucher à d'autres autorités possédant la fonction d'instruction. Les organes d'instruction du parquet ont été transformés en « comité d'instruction auprès de la Procurature générale de la Fédération de Russie » dont le chef est nommé par la Chambre haute du Parlement (le Conseil de la Fédération), c'est-à-dire de la même façon que le Procureur général lui-même. Suite aux modifications importantes apportées au CPP par cette même loi du 5 juin 2007, le parquet a perdu dans une large mesure les fonctions de contrôle sur l'instruction préparatoire, car la majorité de ses compétences en la matière ont été transférées vers les chefs hiérarchiques des agents d'instruction au sein du "comité d'instruction ». Cependant, comme on le sait maintenant, il ne s'agissait que d'une réforme transitoire, car très rapidement le législateur a adopté la nouvelle loi «Sur le comité d'instruction de la Fédération de Russie », qui est entrée en vigueur le I 5 janvier 20 | I et a complétement coupé le lien entre le Ministère public (Procurature russe) et le nouveau Comité d'instruction, devenu absolument autonome vis-à-vis le parquet. Actuellement, ce sont des agents d'instruction du Comité d'instruction qui instruisent en Russie les affaires pénales les plus importantes (le reste de l'instruction ressort de la compétence du ministère de l'intérieur, en ce qui concerne les infractions de droit commun, et du Service de sécurité d'Etat, en ce qui concerne le terrorisme et les autres infractions les plus dangereuses contre la sécurité de l'Etat). De là il résulte que l'instruction russe reste non-judiciaire et les agents d'instruction appartenant aux différents organes d'instruction, y compris le nouveau Comité d'instruction, n'ont pas été transformés en juges d'instruction au cours des réformes russes post-soviétiques. 
En second lieu, même en France, l'évolution récente de l'instruction préparatoire et notamment l'introduction en 2000 du juge des libertés et de la détention qui a récupéré la fonction du juge d'instruction de décider du placement en détention provisoire (par exemple, MATHIAS, p. I33- 135), montre que dans le droit contemporain, le cumul des fonctions de l'instruction et de la protection des libertés et droits fondamentaux n'est plus viconsidéré comme souhaitable. En tout cas en ce qui concerne le droit à la liberté, peutêtre le plus important des droits qui peuvent être limité dans les phases préparatoires du procès pénal, sans oublier à ce sujet qu'en Europe cette matière depuis longtemps «relève de l'ordre public au sein du Conseil de l'Europe» (RENUCCl, p. 79).

En conséquence de ces trois éléments, à savoir que les phases préparatoires du procès pénal russe restent non-judiciaires (non seulement l'enquête, mais également l'instruction), que la restauration du système classique du juge d'instruction n'apparaît pas probable dans le contexte national et comparé et compte tenu des contraintes constitutionnelles, l'intervention d'un juge extérieur à l'instruction pour protéger les libertés et droits fondamentaux dans les phases préparatoires du procès pénal semble être une nécessité. II s'agit alors du modèle du contrôle judiciare, pour reprendre la terminologie de la doctrine russe, c'est-à-dire du contrôle effectué par le juge sur l'instruction préparatoire et l'enquête. Ce contrôle se présente sous deux formes: un contrôle a priori, lorsque le juge a le pouvoir exclusif pour décider de certains actes limitant des libertés et droits fondamentaux, tels que, par exemple, la détention provisoire, la perquisition au domicile ou l'interception des écoutes téléphoniques, et le contrôle a posteriori, lorsque les personnes concernées, surtout l'inculpé et la victime, peuvent attaquer devant le juge les actes des autorités d'instruction violant leurs droits au cours des phases préparatoires du procès pénal. Ces deux formes du contrôle judiciaire existent dans le droit russe en vigueur simultanément, réalisées au fur et à mesure pendant les réformes post-soviétiques et codifiées dans le CPP de 2002.

Mais si la nécéssité du contrôle du juge sur l'instruction et l'enquête est considérée comme évidente dans le contexte constitutionnel, il existe un autre problème à résoudre, à savoir la détermination du juge qui doit intervenir dans les phases préparatoires du procès pénal. Et à ce niveau, la polémique reste très vive.

\section{LE JUGE QUI CONTROLE: UNJUGE ORDINAIRE OU UNJUGE SPECIALISE?}

Le nouveau CPP russe de 2002 a opté en faveur du juge ordinaire. Ainsi, l'al. I 3 de l'art. 108 de ce code dispose explicitement que la prise de la décision sur la détention provisoire ne peut pas être confiée à un même juge de façon permanente. II en est de même pour d'autres fonctions judiciaires dans les phases préparatoires du procès pénal, telles que, par exemple, l'autorisation des perquisitions au domicile ou les recours des particuliers contre les actes des agents d'instruction. Et cette compétence peut être attribuée à n'importe quel juge du tribunal concerné. De surcroît, le juge qui a effectué le contrôle dans les phases préparatoires du procès pénal peut ensuite examiner l'affaire 
sur le fond, ce qui n'est pas interdit. Dans certains cas ce sont des juges différents qui procèdent aux phases de l'instruction préparatoire et du jugement, dans certains cas il s'agit du même juge. Cela dépend notamment de la composition du tribunal, du nombre des affaires à examiner, etc. II arrive souvent dans les petits tribunaux que le même juge cumule les fonctions du contrôle sur l'instruction préparatoire et du jugement de l'affaire, dans les grands tribunaux c'est plutôt une exception.

De là résulte que le législateur russe de 2002 a rejeté l'idée d'un juge de l'instruction (une fonction spéciale), une conception qui est actuellement très à la mode dans l'espace post-soviétique (voir les nouveaux CPP de I'Ukraine, du Kazakhstan, etc.) et qui est recommandée par certains ogranismes internationaux (III Expert Forum, p. 33). Cette idée fut pourtant récemment réanimée en Russie par le très influent Conseil présidentiel pour le développement de la société civile et des droits de l'homme, composé de defenseurs des droits de l'homme, journalistes, artistes, magistrats en retraite etc. et présidé par l'ex-représentant de la Russie auprès de I'UNESCO et actuellement conseiller du Président. En octobre 2014, lors de la rencontre du Président V. Poutine avec les membres de ce Conseil, ce dernier a proposé d'introduire en Russie le système des juges de l'instruction, c'est-à-dire des juges spécialisés qui vont effectuer le contrôle sur l'instruction préparatoire, tant a priori (en étudiant dans les phases préparatoires les demandes sur la limitation des libertés et droits fondamentaux) qu'a posteriori (en étudiant les recours contre les actes des autorités d'instruction). Le Président russe ne s'est pas immédiatement exprimé et a demandé, par une lettre officielle, à la Cour Suprême russe d'étudier la question, d'organiser des débats et de présenter avant le 15 mars 2015 son avis sur la réforme de la procédure pénale relative à l'introduction du juge de l'instruction.

Cette proposition a été examinée à différents niveaux, tant officiels que doctrinaux, et a donné lieu à beaucoup de critiques.

Tout d'abord, si en Europe c'est la France qui est le pays-référence pour le modèle de juge d'instruction, en ce qui concerne le modèle de juge de l'instruction, c'est l'Allemagne qui est normalement citée en exemple avec son fameux ermittlungsrichter, définitivement institutionnalisé après la réforme de procédure pénale de 1974. Néanmoins, l'analyse plus ou moins profonde des pratiques allemandes démontre que la particularité du système allemand n'est qu'un mythe. En réalité, ce système est très souple. La décision de créer une spécialisation ou d'attribuer le contrôle sur les actes de la police et du procureur qui portent atteinte aux libertés et droits fondamentaux à un juge ordinaire se prend au niveau de chaque tribunal. De surcroit, dans certains tribunaux, le même juge qui a accompli ces fonctions de ermittlungsrichter peut ensuite examiner l'affaire sur le fond en tant que juge de jugement. De là découle que la différence en la matière entre le droit russe (où le système de juge de l'instruction officiellement n'existe pas) et le droit allemand (où il existe) revêt un caractère surtout mythique et en tout cas beaucoup moins importante qu'on ne le pense souvent, ce qui rend la réforme envisagée peu utile. 
Par ailleurs, en ce qui concerne la Russie, il est d'imaginer comment le système des juges de l'instruction pourrait fonctionner dans les petits tribunaux, très souvent composés de deux ou trois juges, qui examinent très peu d'affaires civiles et pénales, par exemple dans les zones rurales ou quelque part en Sibérie, où la population n'est pas nombreuse. Quelle est la nécéssité pratique d'instaurer là-bas des fonctions judiciaires complémentaires pour diviser les juges de l'instruction et les juges de jugement?

Enfin, il n'est pas évident, en soi, que le juge spécialisé protège mieux les libertés et droits fondamentaux que le juge ordinaire. Pour quelle raison? La réponse est assez obscure, surtout lorsque l'on analyse les résultats de ce type de réformes dans d'autres pays post-soviétiques, où l'introduction des juges de l'instruction n'a pas provoqué de diminution significative du nombre des détentions provisoires ou d'actes d'instruction limitant les droits fondamentaux. A vrai dire, si l'on prend le cas de l'Ukraine ou du Kazakhstan, aucune diminution n'a en fait été perceptible.

Finalement, l'initiative du Conseil présidentiel pour le développement de la société civile et des droits de l'homme sur l'introduction en Russie des juges de l'instruction, après un débat court, mais efficace, a été rejeté. II a fallu attendre le mois d'avril 20 I 5 pour que la Cour Suprême, sans participer directement aux débats, présente son avis négatif sur la réforme proposée. Elle a été très rapidement suivie par le Ministère public (Generalnaya prokuratura). La question a été close, au moins pour l'instant. La compétence du juge ordinaire de contrôler les phases préparatoires du procès pénal et de décider alors sur la limitation des droits fondamentaux de la personne a été préservée dans le droit russe.

\section{DANS QUELLE MESURE LE JUGE DOIT CONNAITRE LE DOSSIER?}

La critique traditionnelle du contrôle effectué par le juge sur le Ministère public et les autorités d'instruction dans les phases préparatoires du procès pénal réside dans le fait que l'intervention du juge est souvent formelle. Elle permet de légitimer les actes d'instruction (les actes d'enquête) ou les mesures de contrainte, mais ne permet pas de protéger réellement les libertés et les droits fondamentaux, car le juge intervenant, pour ainsi dire, de «l'extérieur» ne connaît pas vraiment le dossier pénal et donc ne peut pas apprécier le fondement de la limitation des droits et des libertés. On se souviendra ici d'une jolie phrase lancée dans la doctrine allemande à propos de l'efficacité de l'activité du juge de l'instruction (ermittlunsgrichter). L'un des auteurs a comparé ce juge et son contrôle sur l'enquête à «une ouvreuse de théâtre qui laisse entrer ou non le spectateur sans véritablement connaître le contenu de la pièce que l'on donne» (WALTHER, p. 142).

Il est même possible de constater qu'il s'agit d'une faiblesse évidente, voire naturelle, du système du «contrôle extérieur» effectué par le juge (le juge de l'instruction ou le juge ordinaire, peu importe) dans les phases préparatoires du procès pénal. Autrement dit, il s'agit de la faiblesse du modèle lui-même. 
De là résulte un taux assez élevé, dans tous les pays, des autorisations données par les juges, lorsqu'ils acceptent la demande du Ministère public, de la police ou de l'autorité d'instruction d'appliquer une mesure de contrainte ou un acte limitant les droits constitutionnels, par rapport aux rejets de ces demandes. Par exemple, en Russie, en ce qui concerne la détention provisoire, les juges ne rejettent les demandes qu'environ dans $10 \%$ des cas (dans $90 \%$ ils les acceptent et ordonnent la détention provisoire). Et il s'agit pour la Russie des juges ordinaires. En Ukraine, l'on retrouve quasiment ces mêmes chiffres, qui n'ont pas particulièrement bougé après l'entrée en vigueur en 2012 du nouveau Code de procédure pénale et l'introduction de juges de l'instruction spécialisés. Et lorsque l'on analyse la pratique des perquisitions au domicile, des écoutes téléphoniques, etc., ces chiffres deviennent encore plus significatifs. ${ }^{2}$

Paradoxalement, le système classique français, voire le système classique soviétique, n'était pas confronté à ce genre de problèmes. Le juge d'instruction français mène l'instruction et connaît le dossier en question en profondeur, d'où découlait notamment sa compétence d'ordonner la détention provisoire de l'inculpé (avant la réforme de 2000). Le procureur soviétique, qui se prononçait, dans ce système, sur la détention provisoire et la limitation d'autres droits fondamentaux, effectuait un contrôle total sur le dossier, c'est-à-dire de façon systémique et non pas ponctuelle. Autrement dit, comme le juge d'instruction français, il connaissait réellement le dossier. Et à l'époque, où c'était le procureur qui procédait en Russie (URSS) au contrôle des phases préparatoires du procès pénal, y compris en ce qui concernait l'autorisation des actes constitutionnellement «sensibles», il rejetait les demandes des agents d'instruction plus souvent que les juges actuels, même dans les conditions du système soviétique qui étaient loin d'être satisfaisantes en matière de protection des droits de l'homme. La doctrine ne trouve qu'une seule explication à ce phénomène surprenant: le procureur connaissait le dossier à la différence du juge actuel qui effectue ses fonctions dans les phases préparatoire du procès pénal ad hoc (ponctuellement).

A vrai dire, la justice russe tente de dépasser ces inconvénients posés par le modèle actuel. Ainsi, la Cour suprême russe dans son arrêté d'assemblée plénière du 19 décembre 2013 a posé qu'en se prononçant sur la détention provisoire le juge doit impérativement vérifier le fondement de la suspicion ou de l'inculpation, c'est-à-dire examiner les preuves concernant la participation de la personne à l'infraction. Pourtant, le même arrêté indique que le juge ne peut pas entrer dans les débats sur la culpabilité, car il ne s'agit pas, à ce moment-là, du jugement de l'affaire sur le fond. Comment trouver un équilibre entre ces deux dispositions? Ce n'est pas, semble-t-il, évident. De là découle une quatrième question, plutôt conceptuelle.

\footnotetext{
${ }^{2}$ Voir, par exemple, le cas de l'Armenie, où en 2008 les juges ont examiné 2688 demandes des perquisitions au domicile, dont 12 ont été rejetées. En 2009, ils ont examiné 3431 demandes et en ont rejetées 24 (AVETISSIAN, p. 90 -91).
} 


\section{MECANISME DE PROTECTION OU MECANISME DE LEGITIMATION?}

Pour terminer, il est possible en conclusion en se demandant quel est le fondement conceptuel de la mission du juge dans les phases préparatoires du procès pénal, lorsqu'il intervient de «l'extérieur» pour décider sur la possibilité de la limitation des libertés et droits fondamentaux dans un cas concret. S'agit-il d'un mécanisme, a priori efficace, permettant de protéger les libertés et droits fondamentaux ou en fait d'une mesure purement institutionnelle qui ne permet que de légitimer (de façon juridiquement correcte) l'activité policière (au sens large), activité qui est en soi, c'est-à-dire institutionnellement, imparfaite, voire viciée, mais absolument indispensable pour garantir l'ordre public?

Nos attentes liée à cette intervention judiciaire ne sont-elles pas un peu démesurées, lorsque l'on attend des résultats concrets, sociologiquement visibles, transformés en statistiques positives etc.? Tandis que le rôle réel du juge dans ce cas est en réalité servir d'instrument purement institutionnel permettant de judiciariser certains actes et procédures, qui sont à l'origine purement policiers. Dans ce contexte, les transformations contemporaines relatives à l'augmentation du rôle du juge dans les phases préparatoires du procès pénal se présentent plus comme une transformation de forme que comme transformation de fond.

Laissons cette question ouverte. Quoi qu'il en soit, il ne faut pas croire en un fantasme: l'apparition d'un juge-protecteur des droits permettant de résoudre tous les problèmes de leur éventuelle violation. II faut, semble-t-il, être plus réaliste, sans confondre l'efficacité au sens sociologique du terme et la légitimation institutionnelle au sens purement juridique.

\section{REFERENCES}

III Expert Forum on Criminal Justice for Central Asia: Final Report. 17- I8 June 2010. Dushanbe, Tajikistan. ODIHR OSCE (http://www.osce.org/ru/odihr/8 | | 6l ?download=true).

Arrêté de l'assemblée plenière de la Cour suprême russe n 4 I du 19 décembre 2013 «Sur la pratique de l'application par les juges de la législation relative à la détention provisoire, l'arrêt domiciliaire et le cautionnement». In: Bulletin de la Cour suprême russe. $N^{\circ} 2$. Février 2014.

AVETISSIAN, Asmik. Les problèmes du contrôle judiciare sur les phases préparatoires. Erevan: EGU. 2010.

FOINITSKY, Ivan. Traité de procédure pénale. Vol. II. 3 éd. Saint-Pétersbourg. 1910. 
GUINCHARD, Serge, BUISSON, Jacques. Procédure pénale. Paris: Litec. 2000.

MATHIAS, Éric. Procédure pénale. Paris: Bréal. 2003.

PRADEL, Jean. Droit pénal comparé. 4 éd. Paris: Dalloz. 2016.

RENUCCl, Jean-François. Droit européen des droits de l'homme. Paris: L.G.D.J. 1999.

STROGOVITCH, Mikhail. Procédure pénale. Moscou. 1946.

TROUSSOV, Alexei. La procédure pénale dans le système de la séparation des pouvoirs. In: Revue de l'Université de Moscou. Série I I. Droit. 1994, n 5.

VINCENT, Jean, GUINCHARD, Serge, MONTAGNIER, Gabriel, VARINARD, André. Institutions judiciaires. Organisation. Juridictions. Gens de justice. 6 éd. Paris: Dalloz. 2001 .

WALTHER, Julien. Les procédures d'exception et l'instruction en Allemagne. Le déferlement de «l'exception» et les fragiles digues de l'Etat de droit. In: Procédures pénales d'exception et Droits de l'homme / sous la dir. de J.-P. Céré. Paris: L'Harmattan. 20 I I.

Recebido em: $11 / 11 / 2016$ Aprovado em: 21/12/2016 
